\title{
Escala de abnegación en cuidadores familiares de adultos mayores
}

\author{
Miriam Teresa Domínguez-Guedea ${ }^{1}$ y Rolando Díaz-Loving ${ }^{2}$
}

1 Universidad de Sonora. Dpto. de Psicología y Cs. de la Comunicación, Sonora (México).

2 Universidad Nacional Autónoma de México. Facultad de Psicologí, División de Investigación y Posgrado, México D.F. (México).

\begin{abstract}
Resumen: La literatura sobre cuidadores familiares de adultos mayores señala que la abnegación es un atributo característico en quien asume el rol del cuidado aún a expensas del deterioro de la propia salud física y/o emocional; sin embargo, no se ha desarrollado un instrumento psicométrico que posibilite el estudio de la abnegación en muestras grandes de cuidadores y que genere evidencia que se pueda integrar a modelos comprehensivos del bienestar en cuidadores. Con el marco teórico y empírico de la etnopsicología mexicana, se diseñó una escala de abnegación que se aplicó a dos muestras de cuidadores $(n 1=115 ; n=351)$, mediante un muestreo no probabilístico. Análisis factoriales exploratorios revelaron dos dimensiones que explican $60 \%$ de la varianza del atributo: Auto-modificación para evitar tensiones familiares y Auto-sacrificio para satisfacer necesidades familiares. Análisis factoriales, vía ecuaciones estructurales, confirmaron el modelo de medida sometido, de acuerdo a los siguientes índices de bondad de ajuste: SRMR $=.039$, RMSEA $=.062, \mathrm{CFI}=.971, \mathrm{GFI}=.975, \mathrm{NFI}=.950 \mathrm{La}$ escala mostró propiedades de validez y confiabilidad adecuadas, indicando factores culturalmente relevantes y conceptualmente claros.

Palabras clave: Abnegación; adultos mayores; cuidadores familiares; validación; etnopsicología.
\end{abstract}

Title: Elder's Family Caregiver Abnegation Scale.

Abstract: Literature about family caregivers of the elderly identify abnegation as a main attribute in those who assume the caregiving role. This characteristic comes at the expense of their own physical and/or emotional health; however, no psychometric instruments have been developed that study abnegation within large caregiver samples which can in turn generate evidence that could be integrated into comprehensive models of well-being in caregivers. Aided by the theoretical frame and empirical background of Mexican ethnopsychology, an abnegation scale was designed and applied to two caregivers samples ( $\mathrm{n} 1=115 ; \mathrm{n} 2=351$ ), using non probabilistic sampling. Exploratory factor analysis revealed two dimensions that explained $60 \%$ of test variance: Self-modification to avoid familiar tension and Selfdenial to meet family needs. Factor analysis through structural equation modeling confirmed the submitted measure model, in accordance to the following goodness of fit indexes: SRMR $=.039$, RMSEA $=.062, \mathrm{CFI}=$ $.971, \mathrm{GFI}=.975, \mathrm{NFI}=.950$. The scale showed adequate validity and reliability properties, indicating culturally relevant and conceptually clear factors.

Key words: Abnegation; elder; family caregiver; validation; ethnopsychology.

funge como cuidador principal - o único -. Las demandas que plantea la asistencia al adulto mayor con problemas de salud se suman a las exigidas por los demás roles sociales de quien asume las responsabilidades del cuidado (madre/padre, esposa/o, trabajadora/o, hermana/o, etc.), exacerbando así el desgaste físico, emocional y económico del cuidador (Domínguez-Guedea et al, 2009).

La literatura sobre cuidadores familiares tiene suficientemente registradas las consecuencias negativas de la sobrecarga del trabajo derivado de las tareas del cuidado, indicando que dicha labor aumenta los riesgos ante diversos padecimientos físicos y emocionales, incluyendo sentimientos de soledad, depresión, ansiedad, fatiga, estrés y sobrecarga (Arango-Lasprilla, Lehan, Drew, Moreno, Deng, \& Lemos, 2010; Marziali, McCleary \& Streiner, 2010; López \& Crespo, 2007). Sin embargo, es mucho menor el conocimiento acerca de las motivaciones que los cuidadores tienen para iniciar y mantener su labor a pesar de las dificultades que ello implica (Kita \& Ito, 2013).

En la caracterización de los cuidadores familiares, frecuente se señalan características de afecto, sacrificio y abnegación como atributos que hacen posible la permanencia del cuidador en este rol que exige tanto (Krmpotic \& De Ieso, 2010). De esos aspectos, en especial llaman la atención las diversas consideraciones que se indican en relación a la abnegación, pues ésta ha sido entendida como una herencia social hacia las mujeres (Rojas, 2007), que inhibe su desarrollo personal (Krmpotic \& De Ieso, 2010), pero también es vista como una capacidad o valor que permite afrontar las dificultades del cuidado y como una práctica con consecuencias positivas para quien recibe los cuidados (Ballesteros, 2008; Fernandes, 2003; Gómez, 2007; Luengo, Araneda \& 
López, 2010; Luzardo, Gorini \& Silva, 2006). A pesar de su papel multifacético en la trayectoria del cuidador, no se ha hecho un estudio sistemático sobre el rasgo de abnegación entre familiares que cuidan a un adulto mayor dependiente funcional.

Desde el área de estudios de la etnopsicología mexicana, encabezada por el trabajo teórico y empírico de DíazGuerrero, se puede encontrar un conocimiento sólido sobre el papel de dicho atributo dentro del complejo dialéctico cultura-contracultura a partir de la cual se organiza el comportamiento de los individuos; siendo así, existe abundante evidencia acerca de la abnegación como una característica fundamental en la dinámica de interacciones dentro de entornos sociales, especialmente a nivel familiar (Holtzman, DíazGuerrero \& Swartz, 1975 citados por Díaz-Loving, 2005).

Entendida como la "disposición conductual para que los otros sean antes que uno, o a sacrificarse en servicio de los otros" (Díaz-Guerrero, 1993, p.4), la abnegación es un rasgo de personalidad en el que se concretan premisas históricas socioculturales mexicanas que mandatan la obediencia afilitativa como principal recurso para la vida social, de manera que el comportamiento es guiado más por amor, cariño o amistad, que por poder o auto-afirmación (Díaz-Guerrero, 1996). De esta forma, en nuestra cultura afiliativa la abnegación implica la renuncia voluntaria de los deseos - o derechos - en favor de alguien más, teniendo esta disposición como referencia la integración intergeneracional de concepciones judeocristianas, e inclusive prehispánicas, que valorizan al "autosacrificio de Cristo a favor de los hombres...y también... la figura de Quetzalcóalt que se inmola para renacer como Venus y hacer que sobreviva el hombre dentro del Universo" (Lara-Tapia, Gómez-Alegría \& FuentesMorales, 1993, p. 30).

En este contexto, el rasgo de abnegación puede ser una característica fundamental para entender las circunstancias bajo las cuales los cuidadores asumen y mantienen la responsabilidad de atender a un familiar adulto mayor dependiente funcional, a pesar de lo extenuante que esto puede ser y de las múltiples repercusiones de este patrón de comportamiento para el propio bienestar. Una comprensión de este tipo es necesaria con miras al diseño de programas de apoyo capaces de captar las razones culturales del cuidado, aspecto ausente en las intervenciones dirigidas a cuidadores familiares de mayores dependientes y que, dicho sea de paso, hasta el momento han logrado una efectividad limitada (López \& Crespo, 2007).

Considerando el valioso potencial de analizar a la abnegación como parte de las características de personalidad de los cuidadores familiares en el contexto mexicano, un primer paso en la agenda de investigación al respecto es el de obtener una medida válida y confiable de dicho constructo. En este sentido, el objetivo del presente estudio es relatar el diseño y validación de una escala conformada para medir abnegación entre cuidadores familiares de adultos mayores dependientes funcionales. El estudio tuvo un diseño no experimental, transversal e incluyó dos muestras independientes de participantes; a los datos provistos por los cuidadores de la primera muestra se aplicaron análisis de validación exploratoria y, con los datos de la segunda muestra se desarrollaron análisis confirmatorios.

\section{Método}

\section{Participantes}

Se colectaron datos de dos muestras independientes y con una diferencia de tiempo de levantamiento de aproximadamente un año. Ambas muestras fueron integradas mediante un procedimiento no probabilístico y por conveniencia, teniendo los siguientes criterios de inclusión de participantes: 1) ser familiar y cohabitar con la persona adulta mayor o bien visitarla al menos una vez por semana y; 2) ser responsable de proveer asistencia al adulto mayor al menos una de las actividades indicadas en el índice de dependencia funcional indicado en el apartado de instrumentos. Se definieron como criterios de exclusión el recibir pago por las labores de cuidado, así como aquellos casos en los que la división del ingreso mensual familiar entre el número de usuarios del mismo fuera $\geq \$ 25,000.00$ pesos mexicanos. Se eliminaron los casos que no completaron el 95\% o más, de los ítems del conjunto de instrumentos aplicados.

El tamaño de las dos muestras de cuidadores fue determinado por el requerimiento psicométrico para estudios de validación de instrumentos que exige de cinco a diez participantes por cada uno de los ítems que serán incluidos en análisis de validación exploratoria (Treiblmaier \& Filzmoser, 2010) y de diez a veinte casos por parámetro a estimar en análisis factoriales confirmatorios vía ecuaciones estructurales (Kline, 2011). Siendo así, a continuación se describen las características sociodemográficas de las dos muestras trabajadas.

\section{Muestra 1}

Se colectaron datos de 115 personas que fungen como cuidadores de un adulto mayor dependiente funcional (90.6\% mujeres y $9.4 \%$ hombres). Las edades de los participantes variaron de los 19 a los 82 años (media $=50$; desviación estándar $=13.1)$ y las edades de los adultos mayores que recibieron los cuidados fluctuaron entre los 60 y los 98 años (media $=78$; desviación estándar $=9.4$ ); el tiempo de cuidado del adulto mayor varió de dos meses a los 60 años, registrándose una media de siete años de cuidados. En referencia a la escolaridad de los participantes se obtuvo que $21.7 \%$ tenía como máximo grado de estudios la primaria, $10.4 \%$ secundaria, $38.7 \%$ nivel de preparatoria y $29.2 \%$ tenía estudios de licenciatura o posgrado.

\section{Muestra 2}

Participaron 351 cuidadores familiares, $87.2 \%$ mujeres y $12.8 \%$ hombres. Sus edades fluctuaron entre los 19 y 84 
años $($ media $=49$; desviación estándar $=11.5)$ y las edades de los adultos mayores receptores de cuidados su ubicaron entre los 60 y 102 años (media $=78.7$; desviación estándar $=$ 8.4). El dato de menor tiempo de cuidado al adulto mayor fue de un mes y el dato de la mayor cantidad de años fue de 60 , siendo ocho años el tiempo promedio del cuidado. La mayoría de los cuidadores tuvo como máximo grado de estudios la preparatoria $(32.1 \%)$, seguida por los estudios de licenciatura/posgrado $(29.9 \%)$, después secundaria $(20.9 \%)$ y $17.1 \%$ de los cuidadores tuvo un nivel de escolaridad de primaria o no cursó estudios de educación formal.

\section{Instrumentos}

\section{Muestra 1}

Se diseñó la Escala de Abnegación en Cuidadores Familiares de Adultos mayores (EAb-CFAM), tomando como referencia la Escala de Abnegación validada por AvendañoSandoval (1994), así como la revisión de literatura especializada (e.g. Díaz-Guerrero, 1993; 1996; 2007; Flores, DíazLoving, Guzmán, Bárcenas \& Godoy, 1992; Flores \& Aguilar, 1998). A continuación se describe el procedimiento seguido.

La Escala de Abnegación de Avendaño-Sandoval consta de 20 reactivos distribuidos en tres factores que en conjunto explican $21.1 \%$ de la varianza del constructo; los factores son: abnegación centrada en la familia, abnegación centrada en la conducta social y abnegación sensitiva o cautela. Dado que el interés por el nuevo instrumento a diseñar era su uso entre cuidadores familiares, la dimensión de abnegaciónconducta social (que refiere al comportamiento fuera de la dinámica familiar), fue desconsiderada en este estudio y se abarcó sólo la disposición hacia la abnegación en la familia. Por otro lado, sí se contempló la dimensión sensitiva/cautela respecto a la comunicación no asertiva/pasiva en la familia. Además se dio énfasis al eje teórico de la abnegación que contextualiza al atributo como un reflejo de la obediencia afilitativa del mexicano que, contrario a la confrontación, busca disminuir dificultades, incomodidad o conflictos con los demás, situación bastante común en el rol del cuidado.

Se realizó el plan de prueba con base en tres dimensiones del constructo de abnegación (abnegación centrada en la familia, comunicación no asertiva y disposición para complacer), generando quince reactivos que, si bien tuvieron como referencia las enunciaciones de la escala de AvendañoSandoval (1994), al final del proceso resultaron diferentes pues se incluyeron frases y palabras comprensibles de acuerdo al lenguaje cotidiano en la localidad, así como contenidos señalados por cuidadores en estudios cualitativos previos (Domínguez-Guedea et al., 2009). Todos los ítems fueron redactados en formato tipo Likert con cuatro opciones de respuesta $(1=$ nunca; $2=$ casi nunca; $3=$ casi siempre; $4=$ siempre) que refieren la frecuencia con la cual el respondiente estima comportarse de la forma indicada en el reactivo, específicamente en situaciones familiares.
La versión preliminar de la EAb-CFAM fue revisada por cinco expertos del área de la psicología, psicometría y profesionales de servicios a adultos mayores, además de ser piloteada en personas de la población general que comparten las características socio-económicas y de escolaridad estimadas de la muestra de interés; el jueceo indicó la pertinencia de los reactivos elaborados para medir abnegación y se sugirieron mejoras tales como simplificación de contenidos en ítems específicos; después de operar los ajustes sugeridos por los jueces, el pilotaje mostró la pertinencia del número de ítems para ser respondidos fluidamente y sin dificultad para su comprensión. Con las apreciaciones indicadas en el análisis teórico y semántico, se realizaron los ajustes correspondientes y se aplicó el instrumento a la primera muestra de cuidadores.

Por otro lado, para medir el nivel de dependencia funcional del adulto mayor receptor del cuidado de los participantes de la muestra 1 y la muestra 2, se aplicó un formulario que integró el Barthel Index (Mahoney \& Barthel, 1965) y el Instrumental Activities of Daily Living Scale (Lawton \& Brody, 1969) cuyas versiones originales fueron modificadas para los fines de este estudio; además se agregaron reactivos sobre la capacidad del adulto mayor para comunicarse y mantener relaciones sociales. El formulario resultante constó de 30 preguntas que valoran el nivel de ayuda que requiere el adulto mayor para desempeñar las actividades básicas, instrumentales y de comunicación; el instrumento es respondido por el cuidador de acuerdo las siguientes opciones de respuesta: 1) no necesita ayuda; 2) lo hace con ayuda de alguien; 3) depende totalmente de alguien.

Se aplicó también una ficha de datos sociodemográficos y familiares del respondiente, diseñada para los fines de este estudio.

\section{Muestra 2}

Los instrumentos aplicados a la segunda muestra de cuidadores fueron la ficha de datos sociodemográficos y el instrumento para la valoración de la dependencia funcional señalados anteriormente, así como la versión de la EAbCFAM resultante de la validación exploratoria (ver sección de Resultados), en la cual se redujo a doce el número de reactivos a ser aplicados a la segunda muestra de cuidadores.

\section{Procedimiento}

El protocolo general del cual se deriva este reporte fue sometido al Comité de Bioética e Investigación del Departamento de Medicina de la Universidad de Sonora, recibiendo un dictamen favorable en la calidad de investigación con riesgo mínimo.

Las dos muestras de este estudio fueron contactadas a través de instituciones públicas y organizaciones de la sociedad civil que prestan servicios a adultos mayores, también se identificaron cuidadores mediante indicaciones personales. De esas organizaciones, las que contaban con un comité de 
ética formalmente establecido, nuevamente se revisó y aprobó el proyecto; con la anuencia de las autoridades se entró en contacto con los familiares de los adultos mayores que reciben los servicios, y de acuerdo a lo establecido en la Carta de Consentimiento Informado, se explicaron los objetivos del proyecto y su proceder general, invitándoles a participar de manera voluntaria.

En el caso de los contactos hechos por indicaciones personales, se localizó a los participantes potenciales e igualmente se les explicó las características del estudio, con base en el contenido de la Carta de Consentimiento Informado, convocando la participación voluntaria.

Con los cuidadores que aceptaron tomar parte del estudio se programaron visitas domiciliarias para colectar el total de la información. Dado que a la par de los instrumentos de este estudio, se aplicaron otros como parte del proyecto de investigación general del cual se deriva el presente, fueron necesarias de dos a cinco sesiones de colecta para cada participante, teniendo una duración promedio de una hora cada una de ellas. Los datos sociodemográficos y los del índice de dependencia funcional fueron levantados con la técnica de entrevista estructurada y la EAb-CFAM fue respondida de manera independiente por los cuidadores que así lo prefirieron y asistidos por el entrevistador por quienes lo solicitaron; el llenado de la escala de abnegación duró en promedio 10 minutos.

\section{Análisis de datos}

\section{Muestra 1}

La ruta de análisis de validación exploratoria de la EAbCFAM fue la siguiente:

(a) Se corrieron análisis preliminares para examinar la distribución del banco de datos y cumplimiento de presupuestos multivariados, atendiendo a procedimientos sugeridos por Tabachnick y Fidell (2007). Se observó la frecuencia de datos perdidos por reactivo y de respondientes que omitieron respuesta al 95\% o más del total de ítems aplicados, valores de asimetría en cada ítem, linealidad de las relaciones entre las variables (mediante scatterplots), presencia de multicolinealidad, casos discrepantes multivariados (outliers) mediante los valores de distancia de Mabalanobis.

(b) Para revisar la factorabilidad de la matriz de datos se analizaron los valores de la prueba Kaiser - Mayer -Olkin (KMO), el determinante de la matriz y el Test de Esfericidad de Bartlett.

(c) Para definir el número máximo de factores a retener se hizo un análisis paralelo comparando los valores propios de los componentes principales de la muestra empírica con los valores propios obtenidos de una base con datos aleatorios; se utilizó dicho procedimiento dada el reconocimiento de su precisión para determinar el número de factores a retener (Gaskin \& Happell, 2013). (d) Para verificar si la estructura de factores producidos por los elementos de la EAb-CFAM resulta teóricamente congruente, se realizaron análisis factoriales exploratorios, con el método de factorización de ejes principales; se utilizó la rotación promax dada la alta correlación observada entre los dos factores. Como valores indicativos de la pertinencia métrica de la estructura factorial exploratoria se revisó el porcentaje de varianza explicada por la solución, así como las saturaciones factoriales de cada ítem y sus valores de comunalidad; se obtuvieron los valores de Alfa de Cronbach para determinar la consistencia interna de los factores resultantes así como de la escala completa.

Todos estos procedimientos se realizaron con el uso del software IBM/SPSS versión 21; en el análisis paralelo también se utilizó el software libre RANEIGEN desarrollado por Enzmann (1997).

\section{Muestra 2}

Los análisis aplicados a las respuestas de los participantes de la muestra 2 tuvieron como propósito examinar, y en su caso confirmar la presencia de las dimensiones en el atributo de abnegación, operacionalizado con los ítems de la EAbCFAM.

$\mathrm{Al}$ igual que en la muestra 1 , previo se revisaron las distribuciones de datos y el cumplimiento de presupuestos multivariados (frecuencia de datos perdidos por reactivo, valores de asimetría en cada ítem, linealidad de las relaciones entre las variables, presencia de multicolinealidad y casos discrepantes multivariados (outliers). Para estos procedimientos se utilizó el software IBM/SPSS versión 21.

Posteriormente los datos fueron sometidos al procesamiento de datos politómicos utilizando el modelo Partial Credit Model (PCM), con el software Winsteps versión.3.7 (Linacre, 2006). Los estimadores analizados fueron: (a) valor de dificultad dentro del rango \pm 2.5 para retener un reactivo en la escala (Chávez y Saade, 2009); valores de infit y outfit, considerando aceptables puntuaciones de .5 a 1.5 (GonzálezMontesinos, 2008); (c) valor de discriminación empírica que, de manera deseable debe aproximarse a 1 , sin rebasar el límite inferior de .90 (González-Montesinos, 2008); (d) correlación punto biserial cuyo intervalo aceptable va de .20 a .40 (González-Montesinos, 2008); (e) la frabilidad global que corresponde al valor de alfa, cuyo valor de mayor consistencia es aproximado a 1 (Gregory, 2012). En este estudio no se utilizó el índice de separación de ítems ni el mapa de Wright ya que ambos análisis son indicados en medidas de logro con el propósito de establecer puntos de corte con relación a los niveles de desempeño evaluado, mas no así en el presente instrumento que, siendo una escala de percepción, únicamente tiene la finalidad de describir el rasgo de interés.

Enseguida se llevaron a cabo análisis factoriales confirmatorios de primer orden, vía ecuaciones estructurales, observando los criterios indicados por Kline (2011) en relación 
al ajuste del modelo evaluado; los criterios son: (a) Root Mean Square error of Approximation - RMSEA $\leq 0.08$; (b) Comparative Fit Index - CFI $\geq 0.95$; (c) Standardized Root Mean Square Residual - SRMR $\leq 0.08)$. Para completar la inspección de resultados se observó la Razón chicuadrada $\left(\chi^{2} / \mathrm{gl}\right)$ cuyo valor debe ser $\leq 3$ (Ruiz, Pardo \& San Martin, 2010). Para esto, se utilizó el software IBM/SPSS/AMOS versión 20.

Para determinar si las puntuaciones de los factores de abnegación varían en función de aspectos sociodemográficos y familiares, se realizaron pruebas t de Student y análisis de varianza. Se utilizó el software IBM/SPSS/AMOS versión 20 .

\section{Resultados}

\section{Análisis de los datos de la muestra 1}

En los análisis previos a los de la validación exploratoria, se identificó que ninguno de los ítems a validar tenía un porcentaje de valores perdidos mayor a 5\%, descartando así la posibilidad de la improcedencia del formato bajo el cual los ítems fueron presentados. Sin embargo se identificaron seis respondientes cuyo porcentaje de omisión de respuestas al total de reactivos fue $\geq 95 \%$, mismos que fueron de los análisis, acatando así el criterio de eliminación de participantes.

Dados los valores de asimetría menores a \pm 1 del conjunto de distribuciones, se concluyó que los ítems no se apartaban drásticamente de la normalidad, con excepción del ítem 3 Me gusta hacer más de lo que puedo si es por mi familia, que reveló un valor de -1.13. Enseguida se verificó la linealidad de las relaciones entre las variables mediante scatterplots encontrándose sólo ligeras distorsiones en la concentración de puntos en las gráficas de la relación del ítem 3 con dos reactivos cuyo valor de asimetría fue $\geq .60$. A pesar de que la distribución del reactivo 3 no se reveló totalmente conveniente en términos de su normalidad y relación lineal, dada su contribución teórica para el constructo se optó conservarlo en los procedimientos siguientes y observar con cautela su desempeño dentro del conjunto de análisis de validación.

Por otro lado, se asumió la ausencia de multicolinealidad, pues ninguno de los ítems obtuvo valores de tolerancia $\leq .30$, indicando que la correlación múltiple cuadrada entre variables no implicaba situaciones de singularidad entre los ítems. Posteriormente se identificaron casos discrepantes multivariados (outliers) mediante los valores de distancia de Mahalanobis; comparando los resultados con una distribución de $\chi^{2}[(15)=$ 30.58; $p \leq .01]$, se encontraron tres casos discrepantes, mismos que se eliminaron de los análisis siguientes. Posteriormente se verificó el número de participantes (106) en relación al número de variables a factorizar (15), encontrándose que para éste estudio se cuenta con siete casos por cada ítem a analizar, cantidad suficiente de acuerdo a los criterios indicados por Treiblmaier y Filzmoser (2010).

Para revisar la factorabilidad de la matriz de datos se obtuvieron tres indicadores, encontrando: a) un valor de ,903 en la prueba Kaiser - Mayer - Olkin (KMO); b) un valor de .001 como determinante de la matriz y; c) un valor de $\chi^{2}=$ $725 ; p \leq .000$ en el Test de Esfericidad de Bartlett. Con las anteriores evidencias se procedió entonces a realizar los análisis correspondientes para evaluar las propiedades psicométricas de la medida de abnegación.

Para determinar el número de factores a retener se hizo un análisis paralelo comparando los valores propios iniciales de los componentes principales con los valores propios obtenidos de una base con datos aleatorios; se utilizó dicho procedimiento dada el reconocimiento de su precisión para determinar el número de factores a retener (Gaskin \& Happell, 2013); ese análisis indicó la pertinencia de extraer como máximo dos factores. Se realizaron entonces análisis factoriales por ejes principales, extrayendo dos factores que explican $60 \%$ de la varianza del constructo abnegación de manera coherente teóricamente y con propiedades psicométricas satisfactorias. Se utilizó la rotación promax dada la alta correlación entre los dos factores. En la Tabla 1 se exponen los resultados.

Tabla 1. Análisis factorial de ejes principales y valores de consistencia interna sobre los ítems de la EAB-CFAM $(n=106)$

\begin{tabular}{|c|c|c|c|}
\hline Contenido del ítem & F1 & $\mathrm{F} 2$ & $\overline{h^{2}}$ \\
\hline $\begin{array}{l}\text { Yo hago las cosas por los demás para que ellos no } \\
\text { se cansen }\end{array}$ & .794 & & .588 \\
\hline $\begin{array}{l}\text { Si alguien....me hace sentir mal...prefiero quedar- } \\
\text { me callada(o) }\end{array}$ & .787 & & .594 \\
\hline $\begin{array}{l}\text { Me da pena negarme cuando me piden que haga } \\
\text { algo }\end{array}$ & .756 & & .531 \\
\hline $\begin{array}{l}\text {...hacerme cargo de las cosas para que los demás } \\
\text { no se incomoden }\end{array}$ & .718 & & .696 \\
\hline $\begin{array}{l}\text { Para que estén contentos... hago lo que a los de- } \\
\text { más les toca hacer }\end{array}$ & .696 & & .485 \\
\hline Pienso que debo darle gusto a mi familia en todo & .596 & & .460 \\
\hline Me cuesta trabajo decir que no a mis familiares & .501 & & .397 \\
\hline ....es necesario hacer mis prioridades a un lado & .474 & & .490 \\
\hline
\end{tabular}
para evitar conflictos....

Atiendo las necesidades de mi familia antes que las mías

Me gusta que mi familia sea primero, antes que yo $\quad .768 \quad .495$ Me gusta hacer más de lo que puedo si es por mi $\quad$ 678 $\quad$.477 familia

Cuido primero la salud de mi familia y después la mía

\begin{tabular}{|c|c|}
\hline Alfa de Cronbach por fator & $.882 \quad .834$ \\
\hline Alfa de Cronbach para la escala completa & .899 \\
\hline Correlación entre factores & .698 \\
\hline Varianza total explicada & $60 \%$ \\
\hline
\end{tabular}

El primer factor integró ocho ítems relativos a un patrón de comportamiento en el que se asumen actividades y responsabilidades e inclusive, se abandonan los derechos y prioridades personales para dar gusto y evitar conflictos o 
incomodidad entre los miembros de la familia; el factor también reúne reactivos característicos de una comunicación pasiva, en la que existe dificultad para expresar los sentimientos propios así como para negarse a peticiones de los familiares. Dados los anteriores indicadores de la abnegación, el factor 1 fue denominado Auto-modificación para evitar de tensiones familiares.

Por otro lado, el segundo componente de la estructura factorial fue nombrado Auto-sacrificio por la familia, pues se conforma por cuatro reactivos que indican una tendencia a dar prioridad a las necesidades, hábitos o deseos de los familiares, posponiendo las propias.

De los quince reactivos analizados, tres fueron eliminados porque sus cargas factoriales fueron $<.329$ en cualquiera de los dos factores; los ítems excluidos fueron: Se poner límite a situaciones que me molestan; A la hora de comer, prefiero que mi familia coma primero y después comer yo; Aunque se me cargue más trabajo, me hago cargo de las cosas si alguien más no puede hacerlas.

\section{Análisis de los datos de la muestra 2}

Previo se revisaron las distribuciones de datos, identificando que: a) 17 participantes cubrieron el criterio de eliminación de casos; b) una vez retirados los folios con datos faltantes de al menos 5\% del total de reactivos, el porcentaje de datos perdidos por variable fue nulo; c) siete respondientes se ubicaron como casos discrepantes multivariados, según el valor de $\chi^{2}[(12)=26.22 ; p \leq .01]$, mismos que fueron cancelados en la muestra; d) no se registraron violaciones francas a la normalidad uni o multivariada, ni problemas de multicoli- nealidad, dados las cifras $\geq .46$ en los valores de tolerancia en todos los reactivos.

Posteriormente se aplicaron análisis Rasch para observar el ajuste de los reactivos a la dimensión correspondiente, de acuerdo a lo indicado en la validación exploratoria (ver Tabla 2). Los resultados revelan detalles que permiten tomar decisiones para efectos de mejorar la medida lograda hasta el momento. Específicamente en la dimensión Automodificación, se advierte que cuatro reactivos tienen al menos un valor desfavorable con respecto al resto de los ítems en alguno de los parámetros estimados con el modelo Rasch (lógits, dificultad, ajuste interno/externo, correlación y discriminación), como por ejemplo, el reactivo Me cuesta trabajo decir que no a mis familiares, que presenta valores de ajuste cercanos al límite superior tolerable (1.2), lo que sugiere posible aleatoriedad en las respuestas al ítem, además su valor de correlación con la puntuación total es claramente más bajo que la de los demás reactivos y su poder de discriminación es también menor comparado con el grupo (.72), indicando su limitada capacidad para distinguir a quienes verdaderamente no presentan el atributo; el reactivo Me gusta hacer más de lo que puedo si es por mi familia, presenta también esas dificultades pero en la dimensión Autosacrificio por la familia.

En consecuencia, se decidió retirar los cinco ítems con menor ajuste, para efectos de garantizar la unidimensionalidad de los dos factores de la EAb-CFAM, situación que no afectó la frabilidad de las medidas pues, después de eliminar cuatro ítems de Auto-modificación, su valor de Alfa se modificó de 0.96 a 0.97 y en la dimensión Auto-sacrificio, el valor de fiabilidad varió de 0.98 a 0.94 .

Tabla 2. Estimación de los parámetros de los ítems de EAB-CFAM

\begin{tabular}{|c|c|c|c|c|c|c|c|}
\hline Factor & Reactivos & Lógits & $\begin{array}{l}\text { INFIT } \\
\text { MNSQ }\end{array}$ & $\begin{array}{c}\text { OUTFIT } \\
\text { MNSQ }\end{array}$ & $\begin{array}{c}\text { PTB } \\
\text { CORR }\end{array}$ & $\begin{array}{l}\text { DISCRIMI- } \\
\text { NACIÓN }\end{array}$ & Alfa $^{a}$ Alfab \\
\hline \multirow{8}{*}{ 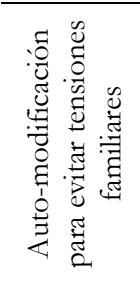 } & Me cuesta trabajo decir que no a mis familiares & -0.15 & 1.18 & 1.30 & 0.58 & 0.72 & \multirow{8}{*}{$0.96 \quad .097$} \\
\hline & Pienso que debo darle gusto a mi familia en todo & -0.01 & 1.04 & 1.09 & 0.64 & 0.95 & \\
\hline & ....mis prioridades a un lado para evitar conflictos.... & -0.01 & 1.07 & 1.06 & 0.62 & 0.90 & \\
\hline & Si alguien....me hace sentir mal... quedarme callada $(\mathrm{o})$ & -0.16 & 1.18 & 1.26 & 0.58 & 0.73 & \\
\hline & ...hacerme cargo de las cosas para que los demás & -0.43 & 0.72 & 0.71 & 0.75 & 1.40 & \\
\hline & Me da pena negarme cuando me piden que haga algo & -0.38 & 0.96 & 0.94 & 0.67 & 1.07 & \\
\hline & Yo hago las cosas por los demás para que ellos no.... & 0.39 & 0.93 & 0.88 & 0.69 & 1.13 & \\
\hline & Para que estén contentos... hago lo que a los demás... & 0.75 & 0.91 & 0.90 & 0.68 & 1.14 & \\
\hline \multirow{4}{*}{ 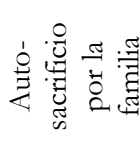 } & Me gusta hacer más de lo que puedo... por mi familia & -1.08 & 1.15 & 1.16 & 0.67 & 0.81 & \multirow{4}{*}{$0.98 \quad 0.94$} \\
\hline & Me gusta que mi familia sea primero, antes que yo & 0.04 & 0.95 & 0.97 & 0.78 & 1.06 & \\
\hline & Atiendo las necesidades de mi familia antes que... & 0.21 & 0.81 & 0.82 & 0.83 & 1.22 & \\
\hline & Cuido primero la salud de mi familia y después la mía & 0.83 & 1.05 & 1.12 & 0.79 & 0.91 & \\
\hline
\end{tabular}

Ante tal evidencia, enseguida se especificó el modelo de medida con siete reactivos a analizar de manera confirmatoria (ver Figura 1).

Se advierten valores satisfactorios en los índices de bondad de ajuste del modelo de medida sometido, así como pesos factoriales significativos de todos los reactivos con su correspondiente latente. Así, se confirma la estructura de la auto-modificación para evitar tensiones familiares y auto- sacrificio por la familia, como las dos dimensiones de la abnegación en cuidadores familiares de adultos mayores. El valor de Hoelter de Critical $\mathrm{N}$ indicó un tamaño de muestra 247 , con un valor de $p \leq .05$ y de 306 con el valor $p \leq$. 01, cifras inferiores a la cantidad de respondientes considerados en el análisis factorial confirmatorio y que consistió en 327 casos. 


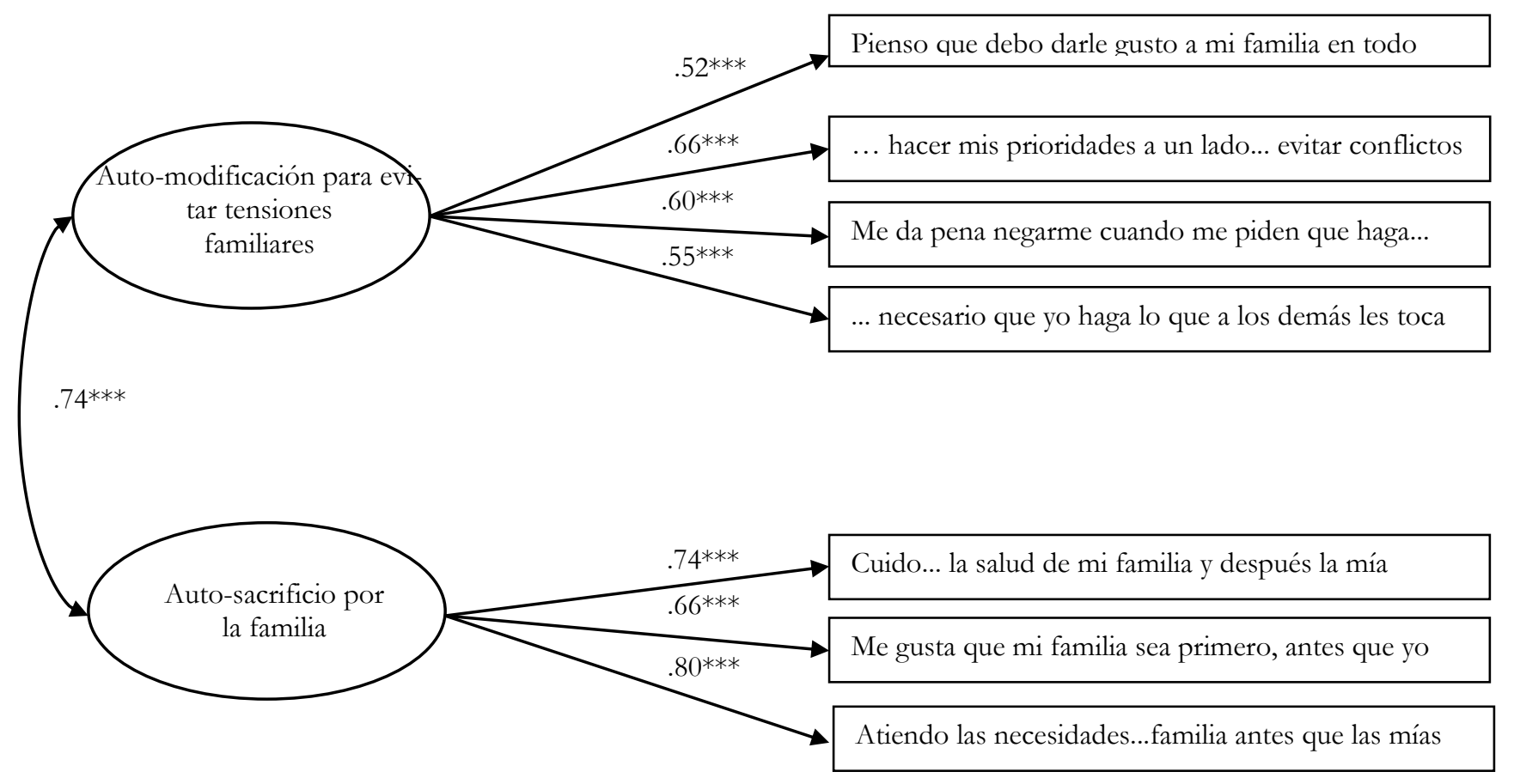

Figura 1. Modelo de análisis factorial confirmatorio de la estructura de la EAB-CFAM $(n=327)$. $\mathrm{SRMR}=.039 ; \mathrm{RMSEA}=.062(.032, .093) ; \chi^{2}(\mathrm{gl})=29.532(13), p=.005 ; \mathrm{CFI}=.971 ; \mathrm{GFI}=.975 ; \mathrm{NFI}=.950 ; * * * p \leq .001$

Mediante un análisis de $t$ de Student para muestras relacionadas, se identificó que la media de puntuaciones de Auto-sacrificio (3.03) es significativamente mayor a la media en Auto-modificación (2.48); los valores resultantes de dicha comparación son: $t=-14.31, \mathrm{gl}=326, p \leq .000$.

Finalmente, para determinar si las puntuaciones de los factores de abnegación varían en función de aspectos sociodemográficos y familiares, se realizaron pruebas t de Student y análisis de varianza que arrojaron resultados significativos sólo para variables de tipo económico y las referidas al contexto del cuidado. Los resultados indican que las puntuaciones de auto-modificación para evitar tensión familiar son significativamente mayores: en cuidadores con menor ingreso familiar mensual, en quienes viven con el mayor receptor de los cuidados, en quienes fungen como cuidador único o principal y entre las personas que han cuidado de otro adulto mayor además del que fue tomado como referencia para estudio.

Tabla 3. Comparación de puntuaciones factoriales de la EAB-CFAM en función de variables sociodemográficas.

\begin{tabular}{|c|c|c|c|c|c|}
\hline \multirow[t]{2}{*}{ Variables sociodemográficas } & \multirow[b]{2}{*}{$N$} & \multicolumn{2}{|c|}{$\begin{array}{l}\text { Auto-modificación para } \\
\text { evitar tensión familiar }\end{array}$} & \multicolumn{2}{|c|}{ Auto-sacrificio por la familia } \\
\hline & & Media & Prueba $t(\mathrm{gl})$ & Media & Prueba $t(\mathrm{gl})$ \\
\hline $\begin{array}{l}\text { Ingreso familiar mensual } \\
\text { Hasta } \$ 9,000 \text { pesos mexicanos } \\
\text { Más de } \$ 9,000 \text { pesos mexicanos }\end{array}$ & $\begin{array}{l}195 \\
129\end{array}$ & $\begin{array}{l}2.74 \\
2.50\end{array}$ & $3.128(244) * *$ & $\begin{array}{l}3.20 \\
3.00\end{array}$ & $2.11(322) *$ \\
\hline $\begin{array}{l}\text { Vive con el adulto mayor } \\
\text { No } \\
\text { Sí }\end{array}$ & $\begin{array}{l}134 \\
192\end{array}$ & $\begin{array}{l}2.52 \\
2.73\end{array}$ & $-2.59(250.9)^{*}$ & $\begin{array}{l}3.07 \\
3.17\end{array}$ & $-1.34(324)$ \\
\hline $\begin{array}{l}\text { Tipo de cuidador } \\
\text { Secundario o co-cuidador } \\
\text { Único o principal }\end{array}$ & $\begin{array}{l}114 \\
188\end{array}$ & $\begin{array}{l}2.40 \\
2.75\end{array}$ & $-4.84(300) * * *$ & $\begin{array}{l}2.97 \\
3.20\end{array}$ & $-2.95(300)^{* *}$ \\
\hline $\begin{array}{l}\text { Ha cuidado a otro adulto mayor } \\
\text { No } \\
\text { Sí }\end{array}$ & $\begin{array}{l}125 \\
201\end{array}$ & $\begin{array}{l}2.54 \\
2.71\end{array}$ & $-2.16(324) *$ & $\begin{array}{l}3.10 \\
3.17\end{array}$ & $-1.49(226)$ \\
\hline
\end{tabular}

${ }^{*} p \leq .05 ;{ }^{* *} p \leq .01 ; * * * \leq .001$ 


\section{Discusión}

Es inminente el aumento del número de adultos mayores y en consecuencia, de las enfermedades típicas de la tercera edad que se caracterizan por la pluripatología y tendencia incapacitante; en estas circunstancias, los años de vida que han ganado los adultos mayores mexicanos suelen acompañarse de un mayor período de dependencia funcional. Pero en esta alerta demográfica y epidemiológica ha pasado inadvertida la figura de la persona que provee los cuidados cotidianos al adulto mayor dependiente funcional y que, en la gran mayoría de los casos es un familiar.

A diferencia de países anglosajones, europeos y de Brasil como caso latinoamericano, en México no existe una política pública para apoyar a quienes asumen la responsabilidad de cuidar a su familiar dependiente funcional aún a expensas del deterioro del propia salud, así como tampoco se ha registrado ningún movimiento social que exija al Estado el cumplimiento de su obligación de institucionalizar subsidios para las familias cuidadoras. Con certeza las razones de dicha ausencia son muchas, pero dentro de todas ellas sí es posible pensar que la falta de una demanda y acción social mexicana que visibilice a los cuidadores, refleja una tendencia presente en la dinámica de las relaciones interpersonales de nuestra cultura pues, siendo la abnegación una disposición común y esperada entre las personas, a nadie le sorprende que las tareas del cuidado del adulto mayor se concentren en un familiar y que éste realice sus labores con escaso o nulo apoyo familiar y/o institucional, pero que lo haga siguiendo los mandatos de la obediencia afilitativa, actuando por amor aun sacrificando su propio bienestar.

En este estudio se encontró que la abnegación quedó representada por dos dimensiones coherentes con las características de dicho rasgo en población general, pero también pertinentes al rol de cuidador en lo particular; los factores son: 1) auto-modificación para evitar tensiones familiares y, 2) auto-sacrificio por la familia.

$\mathrm{Al}$ discutir sobre la abnegación como un factor cardinal en la personalidad de los mexicanos, Díaz-Guerrero (1993) señala que la auto-modificación versus la autoafirmación es uno de los aspectos que forman parte de la tendencia del mexicano en cuanto a "la negación de su Yo y de sus necesidades a favor de los demás y de la sociedad” (p. 3). DíazGuerrero (1982, citado en Flores et al. 1992) también indica que la abnegación es, de hecho, un estilo de confrontación pasiva para resolver problemas. En este contexto conceptual, resulta relevante el factor "auto-modificación para evitar conflictos familiares" pues integra reactivos que plantean el hacer cosas para dar gusto y evitar molestias o discusiones en la familia, así como comportamientos propios de la comunicación no asertiva, en la cual no se externan sentimientos personales y se tiene dificultad para defender los derechos.

Empíricamente este factor es comprensible ya los cuidadores familiares generalmente tienen diferentes roles sociales al interior de la propia familia, fungiendo paralelamente co- mo hija/o del adulto mayor, esposa/o, madre/padre, etc.; de esta manera, los cuidadores se ven exigidos también por una diversidad de demandas para lograr relacionarse de manera equilibrada en la compleja dinámica familiar. Además, en un contexto en el que la autoafirmación es socialmente amonestada más que valorizada, al parecer los cuidadores recurren a una comunicación pasiva y desisten de afirmar los propios derechos y prioridades, inhiben la expresión de necesidades o emociones y prefieren modificar el propio comportamiento antes que intentar lograr cambios en el entramado familiar.

El factor Auto-modificación para evitar tensiones familiares es coherente con el factor Abnegación sensitiva o cautela reportado en población general por Avendaño-Sandoval (1994) y confirmado por Avendaño-Sandoval, DíazGuerrero y Reyes-Lagunes (1997), en donde el factor refiere ítems sobre la dificultad para reclamar, poner límites a las cosas y situaciones, apena decir no y ante las provocaciones verbales se opta por callar.

Por su parte, el factor Auto-sacrificio por la familia, trata situaciones en las que el cuidador escoge atender, cuidar y hacer las cosas primero por los miembros de la familia y después atender las necesidades personales. Díaz-Guerrero (1996; 2007) plantea que en México la institución social más importante es la familia y que la mayoría de los mexicanos conciben al nosotros, antes que el yo, a la familia, antes que a las individualidades que la integran. Así, en un entorno colectivista, en el que el proceso de socialización prioriza la armonía familiar y el no abnegarse quebranta el mandato cultural, molesta, ofende o provoca violencia en el otro (Díaz-Loving, 2008), es entendible que el cuidador anteponga el bienestar familiar al propio, condescienda - sea por bondad o necesidad - y satisfaga primero las necesidades de sus familiares y después las suyas.

Dicho factor es equivalente al factor Abnegación centrada en la familia, identificado en población general mexicana por Avendaño-Sandoval (1994) y ratificado por AvendañoSandoval et al. (1997), que aborda situaciones en las que las personas atienden a la familia a pesar de estar cansadas, cuidan el sueño de la familia, trabajan más por la familia, ofrecen la mejor comida para la familia y el primer lugar lo conceden a la familia.

Siendo así, los resultados de esta investigación dan pauta para identificar dos claras razones por las cuales los cuidadores familiares mexicanos se abniegan: para evitar conflictos y procurar el bienestar familiar. En efecto, cuidar del adulto mayor enfermo, asumiendo la carga de trabajo y responsabilidades que debiera estar compartida por el resto de los familiares, así como continuar velando por el resto de sus seres queridos, es la dinámica y experiencia documentada por la literatura sobre cuidadores familiares (Ballesteros, 2008; Fernandes, 2003; Gómez, 2007; Krmpotic \& De Ieso, 2010; Luengo y cols., 2010; Luzardo y cols., 2006).

Los dos factores de la Escala de Abnegación en Cuidadores Familiares de Adultos Mayores exhibieron resultados psicométricos robustos en análisis exploratorios y confirma- 
torios; al identificar que el modelo de medida sometido a confirmación representa con verosimilitud la estructura teórica y empírica de la escala, se tiene evidencia de la validez de constructo de la EAB-CFAM. Cabe destacar que, aún y cuando se retiraron reactivos para garantizar la unidimensionalidad de los componentes de la abnegación, los análisis mostraron medidas consolidadas en términos del apego a los criterios de ajuste del modelo Rasch y ecuaciones estructurales para la medición de variables latentes.

En la segunda muestra de cuidadores consultados, se observó que no existen diferencias estadísticamente significativas en los factores de abnegación en cuanto al sexo del cuidador, ni del adulto mayor, ni en relación a las edades. Dicho resultado se contrapone a lo indicado por la literatura etnopsicológica mexicana que señala que el rasgo de abnegación es más intenso en hombres que en mujeres y en personas con edad más avanzada versus los más jóvenes (DíazGuerrero, 2007), no obstante se debe aclarar que eso se refiere a población general. En este estudio, las personas comparten una característica que las distingue de la población abierta y es que, el hecho de asumir el cuidado de un adulto mayor dependiente funcional probablemente ya sea una condición que atenúa diferencias debidas al género y características intergeneracionales al interior de la muestra participante.

En donde sí se detectaron resultados significativos fue en los aspectos económicos, encontrando que las personas cuyo ingreso familiar mensual es menor a $\$ 9,000$ mil pesos, reportan mayores puntuaciones en los dos factores de abnegación que los cuidadores que sí tienen un trabajo remunerado y los que tienen un ingreso familiar mensual mayor a $\$ 9,000$ mil pesos. Dicho resultado puede ser interpretado en términos de que las posibilidades económicas restringidas pueden exigir del cuidador mayor recurrencia a la automodificación para mantener la armonía en las relaciones familiares que garanticen, inclusive, la propia subsistencia, siendo necesario condescender ante las necesidades y prioridades de los demás integrantes del grupo familiar.

Por otro lado se identificó que las personas que han tenido la experiencia de cuidar a otro adulto mayor, además del actual, también alcanzaron mayores puntuaciones de Au-

\section{Referencias}

Arango-Lasprilla, J. C., Lehan, T., Drew, A., Moreno, A., Deng, X. y Lemos, M. (2010). Health-related quality of life in caregivers of individuals with dementia from Colombia. American Journal of Alzheimer's Disease and Other Dementias, 25, 556-561. doi:10.1177/1533317510382287

Avendaño-Sandoval, R. (1994). Desarrollo y validación psicométrica de una escala de abnegación para adultos. Una aportación de la etnopsicología mexicana. (Tesis de maestría no publicada). Universidad Nacional Autónoma de México, México.

Avendaño-Sandoval, R., Díaz-Guerrero, R. y Reyes-Lagunes, I. (1997). Validación psicométrica de la segunda escala de abnegación para jóvenes y adultos [Psychometric validation of the second abnegation scale for young persons and adults]. Revista Interamericana de Psicología, 31, 47-56.

Ballesteros, P. (2008). Efectos que el cuidado de un enfermo de alzheimer produce en el cuidador primario. (Tesis de licenciatura no publicada). Universidad Javeriana, Bogotá. to-modificación para evitar tensiones familiares y Autosacrificio por la familia. Estos resultados pueden entenderse en el contexto mismo de la dinámica familiar, pues el haber fungido previamente como cuidador aumenta las probabilidades de que el rasgo de abnegación ya haya sido vivido en la calidad de cuidador y que se haya reforzado ante la experiencia actual; asimismo, el hecho de contar con pareja aumenta la complejidad de relaciones intrafamiliares, escalando las demandas y exigencias ante las cuales, tal vez el cuidador prefiera abnegarse.

En palabras de Díaz-Guerrero (1996, p. 12) "la evidencia acumulada....sugiere fuertemente que la primera respuesta en la gran mayoría de los mexicanos, respuesta semiconsciente, casi automática...es la de abnegarse, de automodificarse ante las situaciones...el abnegarse era una virtud para los aztecas como lo es para el cristianismo"; concordando con el autor, ya es tarea de la psicología entender si la abnegación es adecuada o no en el contexto del análisis de sus consecuencias.

Con la conformación de una medida válida y confiable de abnegación en cuidadores familiares de adultos mayores dependientes funcionales, esta investigación ha dado un primer paso para estudiar el rasgo de la abnegación en muestras amplias de cuidadores, quienes posponen o al menos dividen la atención de sus propias necesidades por atender a las de alguien más. Ahora es indispensable avanzar en el estudio de la abnegación en el marco amplio de las relaciones que establece el cuidador con su familiar adulto mayor así como con los demás integrantes de la familia, analizando si la abnegación se asocia a patrones de comportamientos problemáticos en la dinámica familiar y que eventualmente pueden alterar no sólo el bienestar del cuidador, sino también su relación con el adulto mayor, así como la calidad del cuidado.

Agradecimientos.- Los autores agradecen al Fondo Sectorial para la Educación SEP-CONACYT, por los recursos ministrados al Proyecto 99094 "Evaluación de un modelo estructural de bienestar subjetivo en cuidadores familiares de adultos mayores", del cual se deriva el presente reporte de investigación.

Chávez, C. y Saade, A. (2009). Procedimientos básicos para el análisis de reactivos. México: Centro Nacional de Evaluación para la Educación Superior, A. C.

Díaz-Guerrero, R. (1993). Un factor cardinal en la personalidad de los mexicanos. Revista de Psicología Social y Personalidad, 9(2)1-19.

Díaz-Guerrero, R. (1996). La etnopsicología en México. Revista de Psicología Socialy Personalidad, 12 (1-2), 1-13.

Díaz-Guerrero, R. (2007). Psicología del mexicano 2: Bajo las garras de la cultura. (2da ed.). México: Trillas.

Díaz-Loving, R. (2005). Emergence and contributions of a Latin American indigenous social psychology. International Journal of Psychology, 40, 213 227. doi: $10.1080 / 00207590444000168$

Díaz-Loving, R. (2008). De la psicología universal a las idiosincrasias del mexicano. En R. Díaz-Loving et. al. (Eds.), Etnopsicología mexicana: Siguiendo la buella de Diaz-Guerrero (pp. 44-150). México: Trillas. 
Domínguez-Guedea, M., Arruda, F., Montiel, M., Ochoa, M., Álvarez, G., Valdéz, L. \& Ibarra, E. (2009). Necessidades de apoio social em cuidadores de familiares idosos mexicanos. Psicologia \& Sociedade, 21, 242-249.

Fernandes, C. (2003). Percepção de perdas e ganhos subjetivos entre cuidadores de pacientes atendidos em um programa de assistência domiciliar. (Disertación de maestría no publicada). Universidad de São Paulo, Brasil.

Flores, M., Díaz-Loving, R., Guzmán, P. L., Bárcenas, M. G. y Godoy, P. G. (1992). Asertividad, abnegación y agresividad: Evaluación semántica. La Psicología Social en México, 4, 303-308.

Flores, M. M. y Aguilar, C. B. (1998). Asertividad versus abnegación en una cultura tradicional. La psicología social en México, 8, 150-157.

Gaskin, C. J. y Happell, B. (2013). On exploratory factor analysis: A review of recent evidence, an assessment of current practice, and recommendations for future use. International Journal of Nursing Studies, 51, 511-521. doi:10.1016/ j.ijnurstu.2013.10.005

Gómez, M. M. (2007). Estar ahí, al cuidado de un paciente con demencia. Investigación y Educación en Enfermería, 25, 60-71. Recuperado de http://aprendeenlinea.udea.edu.co/revistas/index.php/iee/index

González-Montesinos, M. J. (2008). El análisis de reactivos con el modelo Rasch. Recuperado de http://www.winsteps.com/a/recursos-offline.pdf

Gregory, R. (2012). Pruebas psicológicas. Historia, principios y aplicaciones (sexta edición). México: Pearson.

Kline, R. (2011). Principles and practice of structural equation modeling. (3rd ed.). New York: The Guilford Press.

Krmpotic, C. S. y De Ieso, L. C. (2010). Los cuidados familiares. Aspectos de la reproducción social a la luz de la desigualdad de género [Os cuidados familiares. Aspectos da reprodução social à luz da desigualdade de gênero]. Revista Katálysis. Florianópolis, 13, 95-101. Recuperado de http://www.katalysis.ufsc.br/conteudo.php?\&sys=bd\&id=1

Lara-Tapia, L., Gómez-Alegría, P. y Fuentes-Morales, R. (1993). Cambios socioculturales en los conceptos de abnegación en la familia mexicana: Un estudio en relación con el cambio social. Revista Mexicana de Psicología, 10, 29-35.

Lawton, M. P. y Brody, E. M. (1969). Assessment of older people: Selfmaintenance instrumental activities of daily living. The Gerontologist, 9, 179-186.
Linacre, J. M. (2006). A user's guide to Winsteps Ministep Rasch-model computer programs. Recuperado de http://www.winsteps.com/

López, J. y Crespo, M. (2007). Intervenciones con cuidadores de familiares mayores dependientes: Una revisión. Psicothema, 19, 72-80. Recuperado de http://www.psicothema.com/

Luengo, C. E., Araneda, P. y López, M. A. (2010). Factores del cuidador familiar que influyen en el cumplimiento de los cuidados básicos del usuario postrado. Index de Enfermería, 19, 14-18. doi:10.4321/S113212962010000100003

Luzardo, A., Gorini, M. y Silva, A.(2006). Caracteristicas de idoso com doença de Alzheimer e seus cuidadores: Uma série de casos em um serviço de neurogeriatria. Texto \& Contexto - Enfermagem, 15, 587-594. doi: 10.1590/S0104-07072006000400006

Mahoney, F. I. y Barthel, D. (1965). Functional evaluation: The Barthel Index. Maryland State Medical Journal, 14, 56-61.

Marziali, E., McCleary, L. y Streiner, D. L. (2010). Evaluation of an assessment battery for estimating dementia caregiver needs for health and social care services. American Journal of Alzheimer's Disease and Other Dementias, 25, 446-454. doi:10.1177/1533317510370958

Rojas, M. (2007). Asociación entre la habilidad de cuidado del cuidador, el tiempo de cuidado y el grado de dependencia del adulto mayor que vive situación de enfermedad crónica, en la cuidad de Girardot. Avances de enfermería, 25(1), 33-45. Recuperado de http://www.enfermeria.unal.edu.co/revista/

Ruiz, M.; Pardo, A. y San Martin, R. (2010). Modelos de ecuaciones estructurales. Papeles del Psicólogo, 31(1), 34-45.Recuperado de http://www.papelesdelpsicologo.es/ pdf/1794.pdf

Tabachnick, B. y Fidell, L. (2007). Using multivariate statistics. (5th ed.). New Jersey: Pearson Education.

Treiblmaier, H. y Filzmoser, P. (2010). Exploratory factor analysis revisited: How robust methods support the detection of hidden multivariate data structures in IS research. Information \& Management, 47, 197-207. doi:10.1016/j.im.2010.02.002

(Artículo recibido: 15-01-2014; revisado: 13-09-2014; aceptado: 23-11-2014) 